www.jmscr.igmpublication.org

Index Copernicus Value: 79.54

ISSN (e)-2347-176x ISSN (p) 2455-0450

crossrefDOI: https://dx.doi.org/10.18535/jmscr/v7i3.106

Journal Of Medical Science And Clinical Research

IGM Publication

An Official Publication of IGM Publication

\title{
Excision of Low Fistula in Ano under Local Anaesthesia
}

\author{
Authors \\ Dr Hitesh Bhatia ${ }^{1 *}$, Dr Priya Bharati ${ }^{2}$, Dr Samta $^{3}$; Dr Sandeep Bhatia ${ }^{4}$ \\ ${ }^{1}$ Senior Resident, Department of Surgery, Government Medical College, Amritsar, Punjab, India \\ ${ }^{2}$ Senior Resident, Department of Medicine, Government Medical College, Amritsar, Punjab, India \\ ${ }^{3}$ Medical Officer Specialist, Department of Dermatology, Civil Hospital Amritsar, Punjab, India \\ ${ }^{4}$ MBBS Intern, Department of Surgery, Government Medical College, Amritsar, Punjab, India \\ *Corresponding Author \\ Dr Hitesh Bhatia
}

Address: Flat No. 19, Registrar Flats, Government Medical College Campus, Amritsar, Punjab, India.

Phone No.9855951599, Email: dr.hiteshbhatia009@gmail.com

\begin{abstract}
Background: More than 95\% of all anorectal abscesses are caused by infections arising in the anal glands that communicate with the anal crypts. Fistula-in-ano in most of cases is a benign condition which causes a considerable agony and interferes with daily quality of life of patients. Most anorectal surgeries are routinely performed under general or regional anaesthesia, but there is a growing evidence of ambulatory day care surgeries under local anaesthesia. In the present study, all cases were done under local anaesthesia with lidocaine $2 \%$ and bupivacaine $0.5 \%$ as local anaesthetic agents.
\end{abstract}

Method: All cases underwent fistulectomy under local anaesthesia and were explained about the interpretation of visual analogue scale. None of the case suffered any lignocaine/bupivacaine toxicity or any systemic complications of anaesthesia.

Results: The average time taken for surgery including the anaesthesia infiltration time was approx 26 minutes. Visual analogue score for pain documented during operation, operative day and postoperative day 1 showed no more than 7, with an average of 3-4 during the procedure.The average duration of hospital stay was approx 2 days in most cases

Conclusion: The overall benefits of early ambulation, good tolerability and fewer anaesthesia complications makes it a safer and reliable method for treating fistula in- ano under local anaesthesia.

Keywords: Fistula In-Ano, Visual analogue score, Local Anaesthesia, PONV.

\section{Introduction}

Fistula is defined as an abnormal communication between two epithelial surfaces.

Fistula-in-ano is an abnormal track which opens deeply in the anal canal or the rectum and peripherally on the skin around the anus. It usually results from an anorectal abscess which bursts spontaneously or is opened inadequately ${ }^{1}$.
More than $95 \%$ of all anorectal abscesses are caused by infections arising in the anal glands that communicate with the anal crypts (cryptoglandular hypothesis).The acute phase of the infection causes an anorectal abscess while the chronic stage is recognized as an anal fistula. ${ }^{2}$ The importance of anal (intramuscular) glands known variously as intramuscular, anal, perianal and 
crypt glands have been recognized in most anorectal pathologies. Usually 6-10 in number, they open into the anal crypts, mostly located in submucosa but also penetrate the internal sphincter. The infection of these glands is responsible for so called cryptitis, ano-rectal abscess and fistulae.

Anatomically, The internal anal sphincter is innervated by sympathetic (L1-L3) and parasympathetic (S2-S4) nerve fibres; both inhibiting sphincter contraction. The external anal sphincter and puborectalis muscles are innervated by the inferior rectal branch of the internal pudendal nerve. Sensory innervation to the anal canal is provided by inferior branch of the pudendal nerve.

Fistulae in ano are commonly divided into two groups:

Low-level fistula-in-ano: Internal opening open into the anal canal below the anorectal ring; those can be laid open with less risk of permanent incontinence.

High-level fistula-in-ano: Internal opening open into the anal canal above the anorectal ring; those can be treated only by staged operations. Park and colleagues ${ }^{3}$ classified fistulae as:

1.Intersphincteric: passing through the internal sphincter via intersphincteric plane to the skin \&is most common type.

2.Transsphincteric: passing through both the internal and external sphincters before exiting to the skin .

3. Suprasphincteric: starting in the intersphincteric plane, passing to a supralevator location, and ultimately tracking between the puborectalis and the levator ani muscles to end in the ischiorectal fossa.

4. Extrasphincteric: described by a supralevator internal opening with a tract, that passes through the entire sphincter mechanism as it exit at the skin.

\section{Goodsall's Rule}

When the external opening lies behind a transverse line passing across the anus or anterior to this line but beyond $3.8 \mathrm{~cm}$ from the anus, the internal opening will be found in the midline posteriorly between the sphincters, the fistulous track being curved. Secondly when the external opening is situated anteriorly to or on the transverse line, but within $3.8 \mathrm{~cm}$ of the anus, the internal opening lies in the same radial line as the external orifice, the fistulous track being straight. One must remember that this is a 'rule' and not a 'law'. There are exceptions to "rules" 4

\section{Clinical Presentation}

A fistula can present as an acute abscess or in a number of ways including;

$\square \square$ Perianal discharge

$\square \square$ Pain

$\square \square$ Swelling

$\square \square$ Bleeding

$\square \square$ Diarrhoea

$\square \square$ Skin excoriation

No specific laboratory studies are required in the diagnosis, Instead physical examination findings remain the mainstay of diagnosis. The examiner should observe the entire perineum. Spontaneous discharge may be apparent via external opening. Digital rectal examination may reveal a fibrous tract or cord beneath the skin. Lateral or posterior induration suggests deep postanal or ischiorectal extension. Anoscopy is usually required to identify the internal opening.

Imaging studies ${ }^{5}$; Radiological studies including fistulography are not performed for routine fistula evaluation. However, they can be helpful when the primary opening is difficult to identify.

\section{Management}

Practice Parameters for Patient established by the Standards Practice Task Force of the American Society of Colon and Rectal Surgeons ${ }^{6}$ for the evaluation and treatment of fistula in-ano.

1) Simple anal fistulas may be treated by fistulotomy or with track debridement and fibrin glue injection.

2) Complex anal fistulas may be treated with endorectal advancement flap closure or with debridement and fibrin glue injection use of a seton and/or staged fistulotomy. 
The goal of treatment is eradication of sepsis without sacrificing continence.

Various treatment modalities are available for fistula in-ano i.e. Fistulotomy (lay open technique $)^{7}$, Fistulectomy ${ }^{8,9}$ and Seton placement ${ }^{10}$.

\section{Anaesthesia}

Fistula-in-ano can be treated surgically under general, spinal as well as under local anaesthesia .Presently in our set-up, fistula was being done under spinal anaesthesia and sensorcaine heavy but in the present study all cases were done under local anaesthesia with lidocaine $2 \%$ and bupivacaine $0.5 \%$ as local anaesthetic agents. Local anaesthetics produce anaesthesia by reversibly binding to and inactivating sodium channels.

Lidocaine (lignocaine, xylocaine) has become one of the most widely used local anaesthetics across the world. In concentrations of $0.5-2 \%$, it produces a rapid onset of intense motor and sensory nerve blockade characterized by a rapid onset of action and intermediate duration of efficacy. Thus suitable for infiltration, block, and surface anaesthesia.

Bupivacaine is a potent agent (commercial preparation concentrations $0.1-0.75 \%$ ) with a slow onset, but prolonged duration of action indicated for local infiltration, peripheral nerve block, sympathetic nerve block, and epidural and caudal blocks.

Normal dose of lignocaine with/without adrenaline ranges from 5 to $7 \mathrm{mg} / \mathrm{kg}$ body wt. with rapid onset and duration of anaesthesia is up to 120 minutes and bupivacaine 2.5 to $3 \mathrm{mg} / \mathrm{kg}$ body wt. with slow onset of action and duration up to 4 hours.

Addition of epinephrine to the local anaesthetic solution may improve safety and allow administration of lower doses of local anaesthetic. Since local anaesthetics are vasodilators, they tend to be absorbed into the bloodstream from the operative field because of vasodilatation of peripheral arterioles. Epinephrine induces vasoconstriction, delaying absorption of the local anaesthetic for longer duration of action at the site of injection increasing the safe dose of local anaesthetic.

\section{Aims and Objectives}

1) To assess the feasibility and tolerability of fistulectomy done under local anaesthesia.

2) To assess the complete excision of the fistula under local anaesthesia.

3) To evaluate the postoperative morbidity, recurrence and complication of fistulectomy done under local anaesthesia

4) To assess the necessity of conversion of local anaesthesia to general/spinal anaesthesia in fistulectomy cases

\section{Material and Methods}

In the present study, all cases were done under local anaesthesia with lidocaine $2 \%$ and bupivacaine $0.5 \%$ as local anaesthetic agents. Detailed general physical examination, systemic examination, local per-rectal and proctological examination of all patients were done and all cases having low fistula in-ano diagnosed clinically or with fistulogram irrespective of age and sex were taken.

Patients with inflammatory bowel disease, Patient with high fistula-in-ano, Malignancy, Sexually transmitted diseases, previous failed treatment with surgery, Anal warts, Patients with significant cardiovascular conditions and pregnant women were not included in the study.

\section{Operative technique}

After taking the full informed consent, all the patients were operated under standard Protocol with local infiltration with a mixture of $30 \mathrm{ml}$ of $2 \%$ lignocaine, $20 \mathrm{ml}$ of $0.5 \%$ bupivacaine, $10 \mathrm{ml}$ of $7.5 \%$ soda bicarbonate diluted with $0.9 \%$ normal saline upto $150 \mathrm{ml}$ of total solution. This solution was prepared immediately before surgery. Local anaesthetic anal canal block was performed in which about $50 \mathrm{ml}$ of the diluted solution was given perianal block, the first two injections were applied bilaterally at 3 and 9 o'clock position, 
$5 \mathrm{~mm}$ from the border of perianal skin, the solution was administered superficially performing the diamond shape. After 1 minute, further four injections were given deep in the four quadrants, $5 \mathrm{~mm}$ from the border of anal opening which Nystrom et $\mathrm{al}^{11}$ described as a perisphincteric deposition of the anaesthetic agent extending up to the levator ani. About $25 \mathrm{ml}$ of solution was given on either side for pudendal nerve blockade using 25G spinal needle. This local anaesthetic solution provided 60-90 minutes of anaesthesia and reduced intraoperative bleeding.

Then after a wait of another 5-7 minutes, surgery was commenced. The area was thoroughly examined and fistulous track was palpated between thumb and finger to have the idea of type of fistula and site of internal opening. Then proctoscopy was done to ascertain the position of internal opening in relation to the ano-rectal ring and different quadrants of anorectum. Methylene blue with hydrogen peroxide was injected through the external opening to delineate the tract. Then a malleable probe was gently inserted through the external opening of fistula to determine the extent and direction of the track. By the aid of finger in the anal canal, the probe tip was bent round or manipulated in such a way that its tip could be brought out at anus. An elliptical incision given around the external opening close to the probe and the whole of fistulous tract was excised with probe in situ.

Any ramifications of the main tract were dealt in the same way. Irregularities on the floor of the wound and any overhanging skin edges were cut away. All the fibrous tissues and scars were also excised so that a healthy raw area was left and the wound packed with lignocaine and betadine impregnated gauze for complete haemostasis. $\mathrm{T}$ bandage dressing was applied.

In postoperative period, all patients were assessed daily for any postoperative pain, bleeding or any other complication and were discharged when patient passed motion and there was no complication. Repeated follow up was done for 2 months.

\section{Results and Analysis}

The results were evaluated in terms of Time taken for surgery (in minutes), Intra-operative pain in term of visual analogue scale (VAS), Postoperative pain on day of surgery(12 hours after surgery), postoperative day 1(24 hours after surgery), Postoperative analgesia (injectable) requirement, Postoperative bleeding (mild, moderate or severe), Postoperative nausea/ vomiting, Hospital stay (in days).

Wound healing, Secondary bleeding, Incontinence of faeces, Anal stenosis, and Recurrence was noted during follow up.

In our present study, 30 patients $(60 \%)$ had the external opening anterior to the transverse line; while 15 patients $(30 \%)$ had posterior opening. 5 patients had multiple openings (upto 3 openings, mixed). Goodsall's law has been found to be accurate in cases of posterior fistulas in 10 patients out of 15 patients i.e., $66.67 \%$. In cases of anterior fistula, the rule has been accurate in $60 \%$ (18 out of 30 patients).

The internal opening could not be identified in 10 of our patients. These patients underwent fistulogram study and those who showed low level anal fistula were included in the study.

The mean operation time was approximately 26.44 minutes with range from 15 to 45 minutes, which included local solution infiltration time. Satisfactory relaxation of the sphincter was confirmed by painless dilation of the anal canal. The analgesia lasted for a few hours, additional anaesthesia for maintenance was not required in a brief duration of operation in most of patients.

In our study the mean VAS score (Graph 1) during operation was found to be 3.96 ranging from 0 to 8 ; during the operation day (12 hours after operation) it was found to be 4.28 with range of 0 to 7 and postoperative day 1(24 hours after operation) mean VAS found to be 1.68 with range from 0 to 4 . The mean VAS score of pain during operation was found to be less than the VAS score on day of operation, which required postoperative analgesia. 


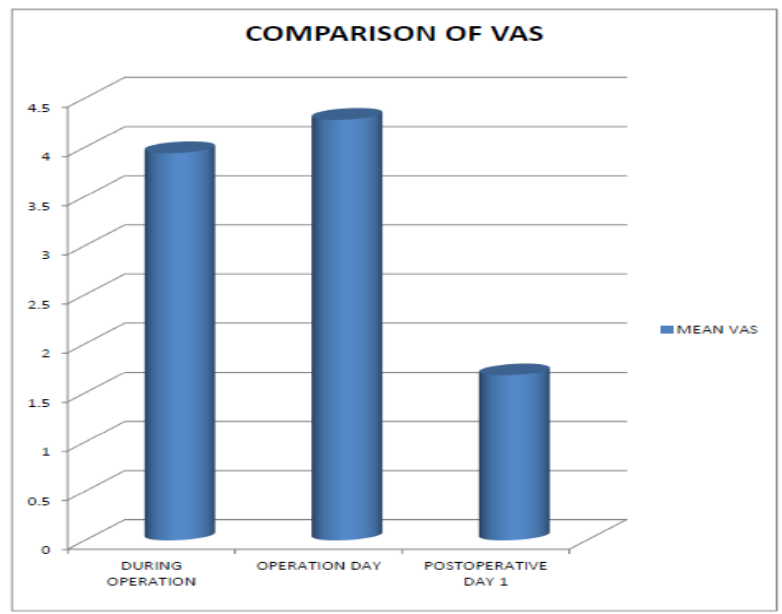

Graph 1: Showing Comparison of Pain VAS score

None of the case in the study showed any signs or symptoms of toxicity due to local anaesthesia nor any case required any conversion to spinal or general anaesthesia. However, patients who unexpectedly complained of pain were given additional doses of solution and injectable analgesia. Injectable postoperative analgesia (NSAIDS) was given in all patients on the operation day in interval of 12 hours. 8 patients required analgesia on 2nd day and 2 patients required on 3rd day. The mean value for duration of injectable analgesia required was 1.2 days.

The incidence of Postoperative Nausea Vomiting (PONV) has remained high and has a major negative impact on patient satisfaction about the overall surgical experience. ${ }^{12}$ PONV (Graph 2) is almost always associated with general anaesthesia and less frequently with regional anaesthesia and least with local anaesthesia. In our study group only 6 patients (12\%) suffered from PONV, mostly during operative day. All cases were managed with one or two doses of antiemetic. In almost all patients, postoperatively oral liquid feed were started and tolerated by the patients as early as 6 hours, however those who suffered PONV delayed feeding or next day feeding was started.
Post Operative nausea/vomiting

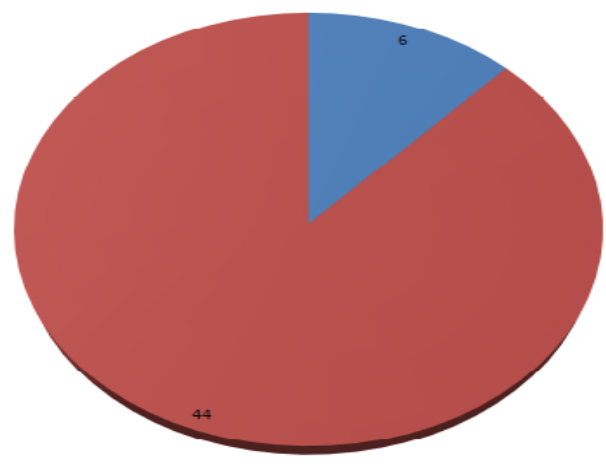

Graph 2: Showing Postoperative Nausea and Vomiting

Postoperative anal pack removal was done next day in almost all cases and dressing and wound were checked for the assessment of bleeding. In 47 cases, mild to minimal soakage was present, so the dressing was removed and the patients were advised for sitz bath in luke warm water 2-3 times a day. In 3 cases (6\%), moderate soakage of dressing due to bleeding was noted, in which anal packing was not removed on the 1st postoperative day and removed on 2nd day. None of the patient had any severe bleeding that required surgical intervention. Retention urine ${ }^{13}$ was seen in 6 patients $(12 \%)$ who required drainage catheterization either with K-90 or foley's catheter. Postoperative fever was noted in 6 patients (12\%) ranging $99-101^{\mathrm{O}} \mathrm{F}$ and was not associated with any rigors or chills. Paracetamol $650 \mathrm{mg}$ once or twice daily controlled the fever. 4 cases developed constipation postoperative which was managed with the use of mild laxative.

Postoperative hospital stay (Graph 3) was found to be average of 2 days. In one case patient was discharged on the same day, 1 day stay found in 24 cases, 2 day in 16 cases, and 3 day in 5 cases, 4 day in 1 case and beyond 5 days in 3 cases. Longer stay in hospital was found to be related to postoperative complications of fever, constipation or moderate bleeding. The average time taken for wound healing in our study was approx 39 days. 
A wound was considered to be healed completely when it was found to be fully epitheliased and dry. The patients in our study were discharged from the ward when the patient passed motion, had no postoperative complication and no bleeding. All patients were advised to have sitz bath in luke warm water with povidine iodine solution regularly 2-3 times daily and then local application of metronidazole-povidine ointment. The minimum time required for complete healing of wound in our study was 22 days and maximum time for complete healing was 65 days. In our study of 50 cases $74 \%$ cases achieved healing in 4-8 weeks of time, $20 \%$ cases in less than 4 weeks.

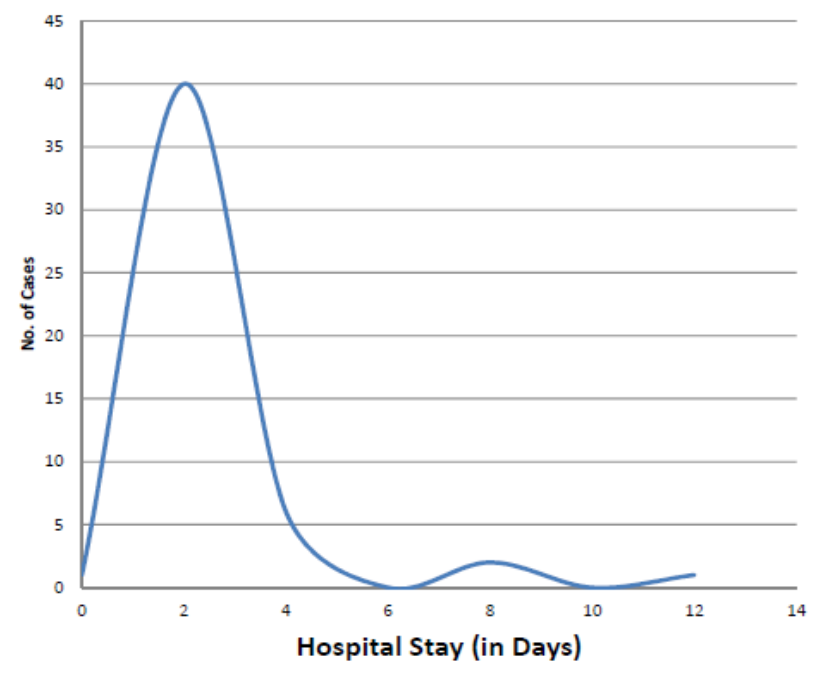

Graph 3: Showing Hospital Stay in Days

Proper hemostasis was achieved during operation with adequate ligation of large bleeding vessels with catgut suture. 2 cases reported minor stenosis of the anal opening managed by regular anal canal dilatation antibiotics and sitz bath. All cases were having low fistula in ano, the damage to anorectal ring was negligible so major incontinence. Major incontinence (defined as any episode of loss of formed stool or persistent leakage of liquid stool occurring more than a week after surgery) was not found in any case. 4 cases reported minor incontinence for few days which was managed conservatively .Recurrence was found to be present in 2 cases after 3 months.

\section{Summary and Conclusions}

Fifty patients diagnosed clinical or radiologically as low fistula in ano were studied prospectively. Following are the conclusions of this study:

- The incidence of fistula was observed in males more than females ( $>2: 1$ ratio), occurring in middle age group (3rd -5 th decade).

- The most common clinical presentation was perianal discharge (96\%) followed by pain and swelling and the average duration of disease was approx 6 months.

- Physical examination includes a digital rectal examination along with proctoscopy helps in identifying the internal opening and level of fistula in majority of cases.

- Fistulogram does not provide any additional information other than identification of internal opening.

- Goodsall's rule was found to be accurate in approximate $60 \%$ in both cases of anterior and posterior external openings.

- The average time taken for surgery including the anaesthesia infiltration time was approx 26 minutes and was not found to be time consuming than other traditional methods.

- Visual analogue score for pain documented during operation, operative day and postoperative day 1 showed no more than 7, with an average of 3-4 during the procedure justifying the tolerability of the technique by the patient.

- All cases underwent complete procedure without any abandonment in any case and whole of the tract was completely removed with achievement of haemostasis, thus proving the feasibility of procedure undertaken.

- Using permissive dosage of local anaesthesia and careful technique of infiltration, none of the case suffered any lignocaine/bupivacaine toxicity or any systemic complications of anaesthesia. 
- Postoperative analgesia was not required for more than 4 days in any patient.

- Oral liquid feed and ambulation was started early in most of the cases.

- Very few cases had early postoperative complications of retention, bleeding, fever or constipation.

- The average duration of hospital stay was approx 2 days in most cases due to early ambulation and very few complications.

- Proper antibiotic coverage, regular daily sitz bath and proper local care of wound, the average healing time by granulation was found to be between $4-8$ weeks $(74 \%$ cases).

- Minor incontinence (8\%), minor stenosis $(4 \%)$ and recurrence (4\%) were among the delayed postoperative complications found.

To summarize, the method of low and simple fistula excision under local anaesthesia is simple, fast, safe and easy to learn with high degree of patient acceptance. It is associated with shorter hospital stay, faster return to full social activities, decreased financial burden on the patient and saves the number of working days lost. Thus, excision of low and simple fistula-in-ano under local anaesthesia is successful and ideal method of treatment.

\section{Bibliography}

1. Parks AG. Pathogenesis and treatment of fistula-in-ano. Br Med J.1961;1:463-9.

2. Abcarian H. Anorectal infection: Abscessfistula. Clinics in colon and rectal surgery. 2011; 24: 14.

3. Parks AG, Stitz RW. The treatment of high fistula in ano. Dis Colon Rectum. 1976; 19: 487-99.

4. Goodsall, D.H. \& Miles, W.E. - AnoRectal Fistula. Diseases of the Anus and Rectum. Longmans, Green \& Co, 1900:92.

5. Sun MR, Smith MP, Kane RA. Current techniques in imaging of fistula in ano: three-dimensional endoanal ultrasound and magnetic resonance imaging. Semin Ultrasound CT MR. Dec 2008; 29(6): 45471.

6. Practice parameters for the treatment of perianal abscess and fistula in ano (Revised). Dis Colon Rectum. 2005;48: 1337-42.

7. Blumetti J, Abcarian A, Quinteros F, Chaudhry V, Prasad L, Abcarian H. Evolution of treatment of fistula in ano. World J Surg. May 2012; 36(5): 1162- 7.

8. Davies, M., Harris, D. and Lohana, P. The surgical management of fistula-in ano in a specialist colo- rectal unit. International Journal of Colorectal Disease. 2008;23: 833-8.

9. Kronborg O. To lay open or excise a fistula-in-ano: a randomized trial. $\mathrm{Br} \mathrm{J}$ Surg. 1985;72: 970.

10. McCourtney JS, Finlay IG. Setons in the surgical management of fistula in ano. $\mathrm{Br} \mathrm{J}$ Surg. Apr 1995; 82(4):448-52.

11. Nystrom PO, Derwinger K, Gerjy R. Local perianal block for anal surgery. Tech Coloproctol. 2004; 8: 23-6.

12. Myles PS, Williams DL, Hendrata M, Anderson H, Weeks AM. Patient satisfaction after anaesthesia and surgery: Results of a prospective survey of 10811 patients. Br J Anesth. 2000; 84: 6-10.

13. Prasad ML, Abearian H. "Urinary retention following operation for benign anorectal diseases", Dis Colon Rectum. 1978; 21: 490-2. 